\title{
EDITORIAL
}

\section{"NON-SPECIFIC" GENITAL INFECTIONS}

Notable advances in the treatment of syphilis and gonorrhoea during the last 10 years, together with a great decline in the incidence of early syphilis since 1946 both in Great Britain and in other countries, have served to focus more attention on the disease, or group of diseases, which is best known under the unsatisfactory name of " Non-specific Urethritis". To the venereologist this is not a new problem, but a familiar and formidable enemy with which he has struggled throughout the years of his specialized work. To the rest of our profession it has the aspect of a newcomer emerging to the light, dimly recognized and very apt to be confused with its close relative, gonorrhoea. The essential features of this condition are well-known to those who work in the subject, and excellent detailed descriptions are available for those who do not. But it may be well to review here certain aspects of the disease which combine to make it one of the important unsolved problems of medical science.

To those familiar with the condition there can be no doubt that non-specific urethritis is a venereal disease, although it lacks statutory recognition as such and will, presumably, continue to do so until the discovery of a specific cause enables the diagnosis to be made with greater certainty. It is a very prevalent disease. The report of the Chief Medical Officer of the Ministry of Health for 1951 (published April, 1953) shows that 10,794 men suffering from this condition attended the public clinics for the first time during that year, as compared with 14,975 new cases of gonorrhoea in the male. Returns of female patients are not available for comparison because the diagnostic standards of this disease in the female are inadequate.

The cause of the condition is, of course, unknown. In recent years speculation has, for the most part, centred in the possibilities of a virus and of organisms of the pleuropneumonia group. Evidence for the former has been based on the finding of inclusion bodies in epithelial cells from the conjunctivae of newly-born babies with conjunctivitis, and from the urethrae and cervical canals of parents of these babies and others. However, it has been rare to find inclusion bodies acceptable to expert opinion from the ordinary run of cases of non-specific urethritis, and the evidence that a virus is the cause in any large proportion of cases is unconvincing. The idea that organisms of the pleuropneumonia group might be responsible has stimulated a good deal of interest. There has been a series of reports of isolation of these organisms from the secretions of a proportion of cases of non-specific urethritis in the male, and from a higher proportion of cases of various genital infections in the female; but there have been some reports, too, of finding these organisms in the urogenital tracts of patients who gave no history of infection and were clinically well. The organisms have certainly been found more often in the infected than in the uninfected, and, such is the variability of this condition and so slight may be its signs, that it is not always easy to establish beyond question who is harbouring infection and who is not. The work of Nicol and.Edward (reported on p. 141 of this issue) adds a careful study to the consideration of this problem. They isolated organisms of the pleuropneumonia group from the secretions of 26 per cent. of 140 men with non-specific urethritis. Similar organisms were found in the urethrae of 11 per cent. of ninety men with no genital abnormality, there being three positive isolations from men who denied sexual intercourse. Cultures from the cervix and vagina of 154 women gave positive results in 77 per cent. of cases, with even higher proportions from those amongst the group who suffered from gonorrhoea or trichomonal vaginitis. The organisms were also isolated from the anal canals of men and women, with or without sign of local infection. Study of serological and biological characteristics of the organisms showed that strains isolated from healthy patients did not differ from those obtained from infected patients. Nicol and Edward therefore conclude that there is no evidence: that the organisms they found play a significant role in genital infection and that they appear to be commensals.

Among the characteristics of this disease which 
have given concern are the relative ineffectiveness of treatment and the tendency to relapse after apparently successful treatment. Many methods of treatment have been recommended and satisfactory results have been claimed for some, but the general experience of even the recent past has not been encouraging. It does seem, however, that some of the reports of results obtained with the newer antibiotics have been more promising and Harkness (p. 134 of this issue) claims a cure rate of 86.5 per cent. in the treatment of non-gonococcal urethritis in the male with oral terramycin, $0.5 \mathrm{~g}$. 6-hrly for 4 days (total 8 g.). The results with other antibiotics were less satisfactory, the equivalent figure for aureomycin being 63 per cent., for streptomycin 39 per cent., and for chloramphenicol 36 per cent. In a second and smaller series of cases for which treatment was prescribed by the method of random selection, the results were similar. Lyall (p. 151 of this issue) claims even better results from a combined treatment consisting of a single deep subcutaneous injection of $1 \mathrm{~g}$. streptomycin, together with $30 \mathrm{~g}$. sulphathiazole given orally in divided doses during 5 days, a form of treatment which has the advantages of being relatively inexpensive and of involving no danger of masking syphilitic infection acquired at the same time.

These are striking claims and if they are substantiated by further experience, uncomplicated non-specific urethritis should present less of a problem in the future. In considering these claims it is well to stress the point, of which the writers are well aware, that it is not easy to determine cure in this type of infection. Claims for any particular method of treatment would be more satisfying if the fate of these patients in the years following treatment could be discovered. Do they, in fact, suffer from further attacks of urethritis which, at the whim of the observer, may be called relapses or reinfections? There is no means of distinguishing between the two, but it is remarkable how often a patient who has once suffered an attack of urethritis is prone to further attacks although his sexual habits remain unchanged.

The complications known to be associated with this infection cover a wide field of bodily disorders, including such dissimilar conditions as prostatitis, epididymitis, pelvic infection in women, conjunctivitis, iridocyclitis, ankylosing spondylitis, arthritis of one or many joints, various cutaneous manifestations, and such combinations of symptoms as the triple syndrome which has been called " Reiter's syndrome". In a thoughtful study of cases of arthritis due to this cause, Ford (p. 123 of this issue) reaches some conclusions and makes some suggestions which may not be acceptable to all who have studied the subject but certainly give food for thought. Ford believes that, as Harkness first suggested, the usual form of arthritis following nonspecific urethritis is identical with that which follows gonorrhoea. The natural history of the disease is characterized by self-limiting attacks lasting from one to 12 months, with a tendency to recurrence in some cases. Permanent damage to joints is not the rule and rheumatoid arthritis is not a sequel, but there are cases with residual deformities of the feet and occasional cases of ankylosing spondylitis. He found that the arthritis was not affected by treatment with antibiotics, but that the administration of ACTH or cortisone in sufficient dosage brought about temporary suppression of the clinical symptoms. From a limited experience with fever therapy, employing comparatively low levels of temperature, he regards it as unproven that fever is an effective treatment. He suggests that the immediate transient improvement which follows fever therapy may be due to temporary adrenal stimulation and that later improvement may well result from the coincidence of spontaneous remission. This is a discouraging thought for those who have advocated and practised fever therapy for years, but the opinion of so careful and unbiased an observer cannot be lightly dismissed. In view of this carefully collected evidence, the claim by Wheatley (p. 162 of this issue) of successful treatment with chloramphenicol of one patient suffering from Reiter's syndrome cannot be accepted as significant.

The fact is that this subject is full of unsolved problems and is beset with reports of unreliable impressions which have too often passed as evidence. Most past forms of treatment have been accepted faute de mieux and have become established through use rather than effectiveness. The key to knowledge is the discovery of the causative organism on which all depends. Here then is the challenge of a neglected major problem, an insidious, highly prevalent, and perhaps incurable disease, which is the source of much more ill-health and unhappiness than is generally suspected. Surely there is the strongest possible case for an organized investigation into this condition, with adequate financial support, in which bacteriologists and clinicians should combine their resources.

\section{REFERENCE}

Ministry of Health (1953). Annual Report of the Chief Medical Officer for the year 1951, covering the period from April 1 1950 , to December 31, 1951. "Part III. On the State of the Public Health ", p. 58. H.M.S.O., London. 